FUTURE INUESTIGATIONS IN ELECTROMAGNETIC NUCLEAR PHYSICS*

\section{E. Hadjimichael}

Center for Theoretical Physics

$1082 \%$

Laboratory for Nuclear Science and Department of Fhysics thechnology

$$
\text { and }
$$

Department of Physics, Fairfield university

Fairfield, Connecticut $05430^{* *}$ G5/23!b

\section{ABSTRACT}

Some basic problems in nuclear physics which may receive positive response from electro- and photonuclear research, are discussed. Future directions for this research are outlined and some specific experimental proposals compiled by a study group under the sponsorship of the Bates (MIT) Users Group are described.

*This work is supported in part through funds provided by the U.S. DEPARTMENT OF ENERGY (DOE) under contract DE-AC02-76ER03069 and the National Science Foundation.

\section{**Permanent Address}

Invited talk presented at the symposium on Perspectives on Electro and Photonuclear Physics, Saclay
October 1980 CTP $\$ 884$

\section{A. Evolution of Photciuclear Physics}

It is possible to follow the evolution of electrons and photons as probes of the nuclear medium by means of a cursory examination of the Proceedings of International Conferences on Photonuclear Reactions.

The origin of photonuclear investigations can be traced to the 1950's and the measurements of nuclear charge distributions. 1 In the early 1960's, the major concerns revolved around measuring the cross sections for $(\gamma, n),(\gamma, p)$ and $\left(e, e^{\prime}\right)$ reactions at the giant resonance region or close to it. From the theoretical point of view the mechanism for giant resonance excitations, and the quasideuteron effect, in addition to testing nuclear models, became subject of rigorous investigations. ${ }^{2}$ Later in the $1960^{\prime} \mathrm{s}$, with the availability of beams of higher energy, new lines of research opened up in terms of pion and vector meson photoproduction, production of isobaric resonances and the nucleon electromagnetis form factors. ${ }^{3}$.

In the early $1970 \mathrm{~s},{ }^{4}$ it is mesonic exchange currents (MEC) and their role in modifying single-body charge and magnetization densities that mark an important stage in photonuclear investigations. In addition, photonuclear and electrunuclear sum rules and their link ro nuclear dynamics were adjed to the constantly expanding list of photonuclear investigations. In the mid $70^{\prime} \mathrm{s}$, we begin to hear about experiments in deep inelastic scattering. 5 Quasi-elastic scattering and coincidence experiments begin to grovide detailed information on electric and magnetic excitations. 
Finally, the proceedings of the conference in Mainz, June 1979 , on Nuclear Physics with Electromagnetic Interactions ${ }^{6}$ provide an almost complete map of the photonuclear field. What is striking about this map is that it shows that virtually all the topics introduced since the origin of the photonuclear investigations in the 1950 's are still under active investigation. A pessimistic interpretation of this circumstance may be that it reflects the inability of the electromagnetic probe to reveal in a definitive way the structure of nuclei and the dynamics that are in operation in the nuclear medium. in optimist on the other hand, would see in the multitude of research lines that have opened up since the '50's and remain open now, a reflection of the versatility of the electromagnetic probe. I tend to side with the latter view and suggest in addition that what is needed now is a great deal of hard work both experimentally in terms of increased accuracy in the data and theoretically in terms of devising imaginative ways to utilize photonuclear data.

Whether we are concerned with the deuteron or with more complex nuclei, the fundamental problem in nuclear physics is the nature of an interacting nucleon pair. We should be reminded of the fact that the models of the $\mathrm{N}-\mathrm{N}$ interaction constructed on the basis of either NN scattering or the deuteron static properties are completely inadequate to explain even the simplest of properties of complex nuclei, as for example the density in the nuclear interior. Whatever success we have had in describing nuclear ground or excited states so far, it has been achieved by means of quasi-empirical forces that are not always obviously related to the NN interaction for free nucleons. Binding obviously changes the nature of the interacting pair. It is of course an accepted fact supported by some experimental evidence that in a nuclear bound state there are at least pions and nucleon resonances in the medium, beșides nucleons. There are however two important questions to be asked and ultimately answered. First, the non-nucleonic components of the nucleus are elements of the NN interaction in the medium; what then is the dependence of the interaction effect on internucleon separation? clearly, the anjwer involves an understanding of both the production and the propagation of non-nucleonic components in the nucleus.

second, how does the structure of the nucleons themselves, e.g., their size, sphericity and the possibility of their having constituent components affect their mutual interaction when they are forced to overlap or at least to exist at very close proximity to each other as it is certainly the case in the interior of nuclei? Are there additional non-nucleonic components that materialize under these conditions?

There is haraly a bettex probe to use in attempting to answer these questions than real photons or virtual photons from electron scattering. We may be reminded again that the entire nuclear volume participates in the charge scattering of electrons and hence the nuclear response function in this scattering, provided it can be separated from magnetic scattering, is an ideal tool to investigate the second question, e.g., the nature of the nucleon pair at very short separation in a bound state. 


\section{B. Future Directions}

Having formulated these two fundamental questions in nuclear dynamics, we must now search for ways to reach for answers. A study group organized under the auspices of the Bates (MI') Users Group ${ }^{7}$ has been attempting for about a year now to chart promising lines for future research in electro- and photonuclear physics so that these and other questions of nuclear dynamics and structure may be answered. Underlying this organizational effort is the rotion that the NN interaction is central to the problems of nuclear physics.

A broad outline of the research proposed by the study group is presented ir. Table 1.

I summarize below some of the objectives to be attaised by this research.

- (e,e!N) experiments which specify the momentum of the electron and nucleon with good energy resolution, determine the transition of the residual nucleus to discrete final states whose wavefunction can then be tested against theory. At high energies for the nucleon, final state interactions do not play an important role.

- Disintegration near threshold (inclusive $\left(e, e^{\prime}\right)$ but with large momentum transfer is sensitive to the high momentum components of the nuclear wavefunction and hence the short-range part of the NN interaction.
- Photo absor on via the quas -istiterun provess can give information on NN correlations in both the initial state when the pair is in the nuclear medium and in the final state.

- Magnetic and electric structure functions of the nucleus are sensitive to meson currents and/or relativistic effects. It is oft $3 n$ useful to separate these two effects.

- The study of isobars will provide answers to the question of how important they are in the description of the NN force.

- Hypernuclei formed via $\left(\gamma, K^{ \pm, 0}\right)$ processes will allow for the study of $\mathrm{N}-\Lambda$ interactions.

- Photoproduction of vector mesons will test coupling constants, the vector meson dominance model and details of the production amplitude.

- Magnetic form factors of very light nuclei give important information on th.e nuclear wave function.

- Parity-violating nuclear processes can be of fundamental importance in testing models of weak interactions, in particular the structure of neutral currents. 
- Whilo the sianature of quark componerts of nucleons is usually thougrit to become apparent at very high enercies, it is imperative to answer the question whether we can understand the short and intermediate range of the NN force and low-energy nuclear processes without explicitly invoking quark degrees of freedom; there are some indications already that inclusion of quark components or, at least, an explicit incorporation of the extended nature of nucleons in nuclear reactions is a necessity.

The study group has solicited proposels on specific electroand photonuclear experiments that may yield the required information on the subjects which, in the opinion of the group, constitute the future of electromagnetic interactions. I shall review below several of these proposals. As it will soon become clear, carrying out some of the proposed work hill require experimental facilities which are not presently available, hence, one of the logical outcomes of this planning effort is that it provides support for building at least one new electron facility of Cw type, suitable for nuclear physics research but capable of delivering energies up to about $2 \mathrm{GeV}$.
SPECIFIC PROPOSALS

\section{NN Correlations by Means of Quasielastic Scattering ${ }^{8}$}

An accurate determination of the entire quasielastic spectrum for ${ }^{2} H\left(e, e^{\prime}\right)$ np is proposed incluaing the tall of the peak that stretches into the region of pion production. With the deuteron as a target, it is probably sufficient to do an $\left(e, e^{\prime} p\right)$ coincidence experiment in the scattering plane ${ }^{9}$ and integrate experimentally over the proton angle in order to obtain the cross section $d^{2} \sigma / d \Omega e d w$, where $w=e n e r g y$ transfer, free from contamination from $\pi$-production events.

A Rosenbluth separation of the charge-scattering part of the cross section $\left(\frac{d^{2} \sigma}{d e^{d w}}\right)$ with an accuracy of $5 q$ will allow us to compare against the standard yukawa NI interaction theory including relativistic effects. A difference of $20 \%$ between experiment and theory can be interpreted with some certainty as evidence that there are serious problems with the Yukawa theory of the NN interaction via meson-exchange at intermediate as well as short range. Discrepancies of smaller magnitude on the other hand, will suggest that we may pursue the meson exchange theory and attempt to determine the short range NN correlations in coordinate space. The integral $\int d w\left(\frac{d^{2} a}{d \Omega_{e} d w}\right)_{c} / \sigma_{\text {mott }}$ is proportioral to the deuteron structure function $s(q){ }^{10}$ which in turn is the Fourier transform of the NN

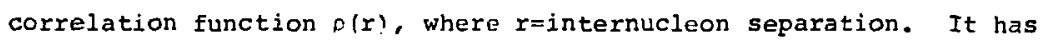
been determined ${ }^{11}$ the for an accurate evaluation of $g(r)$ to about 
$r=0.5 \mathrm{fm}$, we should b.ow $s(\xi)$ with an accuracy of 1 \% up to $q \leq 3 \mathrm{fm}^{-1}$ and 38 for $3 \leqslant q<4.5 \mathrm{Em}^{-1}$. It is precisely this small experimental uncertainty that makes this a very difficult experiment. On the other hand, the value of the information that may be derived from it makes it worth pursuing. It is to be noted that for $q \$ 4.5$, scaling for the nucleon form factors has been shown experimentally to be valid ${ }^{12}$ so that within this range of momentum transfer, we have no substantive uncertainties coming from these quantities.

\section{2. wasurements of Nucleon Electri.c Form Factors}

Should the Yukawa theory of the NN interaction prove viable at all separation distances, then the previous ${ }^{2} H\left(e, e^{\prime} p\right) n$ experiment at higher values of $\mathrm{q}^{2}$ can be used for the determination of nucleon electric form factors beyond the value of $q^{2}$ where scaling seems to break down. For adequate Rosenbluth separations we would need electron beams of energy 3-4 GeV.

A second method for measuring nucleon electric form factors is by scattering longitudinally polarized electrons from hydrogen or deuterium. The information can be extracted from the polarization of the recolling nucleon measured via the asymmetry following a second sattering. The relationship is $\frac{d^{2} \sigma}{d \Omega d \Omega_{2}}=\frac{d \sigma}{d \pi d \Omega_{2}} \int_{0}\left[1+\operatorname{ap}_{x} A_{y} \sin \psi_{2}\right]$

where $a$ is the electron polarization, $\frac{d \sigma}{d \Omega d a} \int_{2} /$ is the double scattering cross section for unpolarized electrons and $\psi_{2}=$ azimuthal angle between the two scatterirg planes; $A_{y}$ is the analyzing power of the second scattering. Finally the polarization

$$
P_{X}=\frac{-2 \sqrt{\tau(1+\tau)} G_{E} G_{M} \tan \frac{1}{2} \theta}{G_{E}^{2}+\tau G_{M}^{2}\left(1+2(1+\tau) \tan ^{2} \dot{\theta} / 2\right)}
$$

is proportional to $G_{E^{*}}$.

It is estimated that 18 statistics will allow the evaluation of $G_{E}$ within 10 .

\section{Low Energy Quasielastic Scattering from ${ }^{3} \mathrm{H},{ }^{3} \mathrm{He},{ }^{4} \mathrm{He}^{14}$}

High momentum components of the nuclear wavefunction contribute primarily to the wings of the broad quasielastic peak. The use of ${ }^{3} \mathrm{H}$ and He isotopes in these experiments is recommended because the central density in these nuclei is sufficiently large to quarantee the presence of a great deal of high momentum components. In addition the possibility that quasielastic cross sections are subject to scaling will be explorcd by means of the same data. The kinematically favorable conditions are high momentum transfer and low energy transfer. ${ }^{15}$

\section{Coincidence Electroproduction from Light Nuclei ${ }^{16}$}

The prototype for these experiments is the reaction $\lambda\left(e, e^{\prime} a^{\prime}\right) \mathrm{X}$, where $a^{\prime}=\pi, p, n,{ }^{2} \mathrm{H},{ }^{3} \mathrm{H},{ }^{3} \mathrm{He}$, etc. kinematically complete experiment is suggested in the momentum region $0.5 \leqslant q^{2} \leqslant 2(\mathrm{GeV} / \mathrm{c})^{2}$ in order to check (a) relativistic aspects of the nuclear wavefunction (b) the $\gamma-a$ ' vertex (c) the degree of compositeness of the spectator (a) the approach to scaling $w w_{2}^{A}\left(w, q^{2}\right) \rightarrow w_{2}^{A}(x)$ of the longitudinal structure function $w_{2}$ of nucleus $A$, and $w=e n e r g y$ transfer. 
5. Tensor polarization in Electron-Deuteron Elastic Scattering ${ }^{17}$

The tensor polarization $t_{2}$ in $e-{ }^{2} \mathrm{H}$ scattering is known to be sensitive to the tensor part of the NN force, and the isoscalar meson exchange currents. The proposed experiment calls for measurements in the range $15<\mathrm{q}^{2}<30 \mathrm{fm}^{-2}$, and would require a high dutyfactor electron accelerator with energy between 1.4 and $2.0 \mathrm{GeV}$ and an average current of $\$ 100 \mathrm{HA}$. One of the immediate results of this experiment will be the precise localization of the minimum in the charge form factor of ${ }^{2} \mathrm{H}$.

\section{6. $(y, \pi N)$ and $\left(e, e^{\prime} \pi N\right)$ Experiments at $1-2 \mathrm{GeV}^{18}$}

There experiments at a high duty factor electron accelerator are for the purpose of investigating the $\pi$-nucleus interaction and in particular the role of the $\Delta$ resonance and the possibility of the presence of a dibaryon resonance. The ${ }^{2} H\left(e, e^{\prime} \pi N\right)$ experiment can measure the production of $\Delta^{\prime} s$ in the nucleus. This information can be utilized in conjunction with $\left(e, e^{\prime} N\right)$ data in heavier nuclei to provide estimates of the presence of $\Delta$ 's in nuclear systems vs. $\Delta '$ a created by photon absorption and ultimately to map the $\Delta$ wavefunction in the nuclear interior.

\section{Photoabscrption Cross Section in the Delta Region by Forward} fingle Electron Scattering ${ }^{19}$

Both single arm $\left(e, e^{\prime}\right)$ and coincidence experiments $\left(e, e^{\prime} \pi\right)$ are proposed for the purpose of measuring the large peak in the region 140-450 Mev due to the $\Delta$ resonance, free of contamination from either quasielastic scattering or radiative effects from scattering below the $\Delta$ regien. For the $\left(e, e^{\prime}\right)$ experiment, the appropriate kinematics are $E_{e}=750 \mathrm{MeV}$ and $\theta_{e}=20^{\circ}$. The $\left(e, e^{\prime} \pi\right)$ experiment becomes quite feasible with a 1008 duty factor beam. The clean photoabsorption spectrum in the Delta region is useful in completing photoabsorption sum rules and checking the validity of the isobarlike model of $\pi$-nuclear reactions.

B. Photo- and Electro-production of $\mathrm{x}^{+, c}$ from Nuclei ${ }^{20}$

A report on recent theoretical calculations of $\left(\gamma, K^{+}\right)$is presented in this conference. ${ }^{20}$ The objective of this work is to extract information on hypernuclear states involving deeply bound $\Lambda$ and $\&$ hyperons. Preliminary estimates show that the favorable kinematical region for hypernuclear studies is in the region of $2 \mathrm{GeV}$ lectron beam energy. Cross sections for the and ${ }^{12} \mathrm{C}$ have been estimated to be of the order of $0.07 \mathrm{\mu b}$ and $0.01 \mathrm{\mu b}$ respectively at this energy. This work takes advantage of the fact that $x^{+, 0}$

is feebly affected by final state interactions.

\section{Measurements of Parity Violatin in the Scattering of Polarized} Electrons from Protons 21

It is proposed that the asymmetry a resulting from the elastic scattering of polarized electrons, from protons, e.g..

$$
A=\frac{\sigma_{2}-\sigma_{R}}{\sigma_{2}+\sigma_{R}}
$$

be measured in the energy region between 0.5 and $2.0 \mathrm{GeV}$. The individual cross sections $\sigma_{L(R)}$ for left and right electron helicity, and hence $A$, are expressed in terms of the coupling constant of the four components of the hadronic weak neutral current. Different 
12

linear combinations of the four couplings are measured at different kinematical points and hence the validity of various nodels of electroweak currents can be investigated.

These nine proposals represent only a small sampl- of the total number that is being compiled. Eurther proposals

pursuant to the goals described in the outline presented earlier, are being prepared by several persons as indicated in the Appendix. Each proposal furnishes the physical justification as well as an account of experimental requirements for the proposed research including energy and beam characteristics and estimates of the accuracy necessary for elucidating the physical ideas of interest.

The study group expects to have its work completed by January, 1981, at which point a volume including both a detailed account of future directions in electromagnetic nuclear physics and the proposal. for experimental investigations which will carry the field along these directions, will become available for distibution.
13

REFERTNCES

1. R. Hofstadter, Flectron Scattering and Nucleon and Nuclear Structure, A. Benjamin, New York, 1963.

2. Proceedings of the Karlsruhe Photonuclear Conference, 1960.

3. International Symposium on Electron and Photon Interactions at High Energy, Hamburg, 1965.

4. International Conference on Photonuclear Reactions and Applications, Asilomar, 1973, ed. B.L. Berman.

5. International sciool on Electro- and Photonuclear Reactions, Erice, Italy, 1976, ed. 5. Costa and C. Schaerf.

6. Nuclear Physics with Electromagnetic Interactions, Mainz, 1979, ed. H. Avenhövel and D. Drechsel.

7. The parel on Future Directions of Electromagnetic Nuclear physics is constituted as follows:

$$
\begin{aligned}
& \text { W. Bertozzi (MIT), Ed Booth (Boston University), } \\
& \text { L. Cardman (Illinois), B. Chertok (American University), } \\
& \text { T.W. Donnelly (MIT), F. Gross (Hilliam and Mary), } \\
& \text { E. Hadjimichael (Fairfield University) S. Hanna } \\
& \text { (Stanford), H. Jackson (Argonne), J. McCarthy (Univ. } \\
& \text { of Virginia), J. O'Connell (NBS), S. Penner (NBS) } \\
& \text { G. Peterson (U. of Massachusetts), P. Stoler (RPI). }
\end{aligned}
$$

8. E. Hadjimichael, Fairfield University.

9. R.J. Budnitz, et al., Phys. Rev. 173, 1357 (1968).

10. K.W. McVoy and L. Van Hove, Phys. Rev. I25, 1034 (1962).

11. G. Bertsch and J. Borysowicz, J. Phys. G. 2, I121 (1976).

12. R. Wilson, Proceedings, Int. Symp. on Elrectron and Phaton Inter. Hamburg, 1965.

13. F. Gross, william and Mary.

14. D. Day and J.5. Mccarthy, University of Virginia.

15. I. Sick, Nucl. Phys. A335, 555 (1980).

16. B. Chertok, American University. 
17. R.3. Holt, Argonne.

18. R.P. Redwine (a) and H.E. Jackson (b), MIT (a) and Argonne (b).

19. J.S. O'Connel1, National Bureau of Standards.

20. A.M. Bernstein, T.W. Donnelly and G.N. Epstein, MIT.

21. P.A. Souder and M.S. Lubell, Yale University.
Table 1: organization of Research in

Electromagnetic Nuclear Physics

I. Nuclear Forces and Nuclear Structure

A. Phenomena Involving Nucleons only

1. NN Correlations

2. Ground state anc Transition Charge and Magnetization Densities

3. Discrete States

4. Giant Resonances

5. Disintegration Near Threshold

6. Quasi-elastic, $\left(e, e^{\prime}\right),\left(e, e^{\prime} N\right),\left(e, e^{\prime} x\right)$

7. Absorption of Real Photons

B. Quasideuteron Effect

B. Phenomena Involving Non-Nucleonic Degrees of Freedom

1. Meson-exchange Currents

2. Isobars and Mesons

4. Relativistic Components

II. The Nucleus as a Laboratory

1. Electroweak Irocesses; Charged and Neutral Currents

1. and Parity Non-conservation

2. Propagation of Unstable Particies

3. Effects of tle Medium on Free Nucleon Phenomena

4. Optical potentials

III. Subnuclear structure

1. Meson and Baryon Spectra

2. Vector meson Production

3. Dibaryons

- Hadronic Form Factors

. Nucleon Polarizability

6. Quark Components, Hidden Color and Bag Models 
APPE:DT:

Present status of the planning effort for future directions of electromagnetic nuclear physics; list of authors of experimental proposals and correspording topics:

N-N Correlations

Ground state and Transition

Charge and Magnetization

Density

Discrete Nuclear states

Giant Resonances

Disintegration Near Threshola

Quasielastic

Real Photons

Quasideuteron

Meson-Exchange Currents

Isobars and Mesons

Hypernuclaj

Charged and Neutral Weak Currents

Propagation of Unstable particles

Vector Meson Pruduction

Hadronic Form Factors
E. Hadjimichael

R, Arnold, W. Bertozzi,

J. Heisenberg, R.J. Holt,

J. Heisenberg, W. Bertozzi

S. Hanna, J. Calarco, L. Cardman

D. Day, J.S. McCarthy

r. Sick, C. Williamson,

B. Chertok

J. Mathews, E. Hayward

J. Lightbody, K. Seth, J. McCarthy

J. Dubach, J. Peterson

R.P. Redwine, H.E. Jackson, J. O'Connell, I. Blomovist, B. Schoch, A.M. Bernstein, T.W. Donnelly

T.W. Donnelly, A.M. Eernstein,

J.N. Epgte in, B.L. Roberts,

J.P. Miller

P.A. Souder, M.S. Lubell

R.P. Redwine, H.E. Jackson

R. Whitney, H. Crannell, J. Noble

F. Gross 